\title{
A Successfully Treated Case of Iliopsoas Abscess Affecting Urinary Tract Infection Caused by Streptococcus sanguis
}

\author{
Nobuhiro Takeuchi,' Kazumasa Emori,, Makoto Yoshitani,, Junichi Soneda,, \\ Hiromichi Naito, ${ }^{2}$ Tetsuya Yumoto, ${ }^{2}$ Kohei Tsukahara, ${ }^{2}$ and Atsunori Nakao ${ }^{2}$
}

\begin{abstract}
Background: Streptococcus sanguis comprises part of the normal flora of the human oral cavity. Although it has been recognized increasingly as an important pathogen of endocarditis, $S$. sanguis is a rare cause of urinary and iliopsoas abscess.

Case Presentation: An 81-year-old male presented to the emergency department with complaints of pain in the right side of his back and gait disturbance. Laboratory results revealed slightly elevated inflammatory markers, moderately elevated renal function, and severe anemia. Computed tomography revealed right hydronephrosis and swollen iliopsoas. Ultrasonography revealed a distended right ureter that was affected by low echoic lesion in the iliopsoas. Iliopsoas abscess was suspected based on imaging results. In addition to administration of an antibacterial antibiotic, percutaneous drainage of the abscess was performed. Cultures of samples taken from the abscess and urine cultures were positive for $S$. sanguis. Fever and inflammatory reaction improved after drainage and antibiotic treatment. Three weeks after drainage, the patient had recovered uneventfully and returned home.

Conclusion: It is important to recognize S. sanguis as a previously unrecognized species of Streptococcus in the etiology of iliopsoas abscess and to be aware of its predisposing factors. Sharing our experience with readers may help clinicians make the proper selection of antimicrobial agents, a key to the successful management of iliopsoas abscess.
\end{abstract}

$\mathbf{I}_{s}$ LIOPSOAS ABSCESS is a rare condition in which an infection spreads from a nearby or distant septic focus to the psoas sheath. It has become uncommon because of the development and implementation of broad-spectrum and highly efficient antibacterial agents. Iliopsoas abscess is divided into primary and secondary types. Primary iliopsoas abscess, defined as an abscess without a secondary infection site, is considered to result from inflammation spread either hematogenously or lymphatically from infection sites. Secondary iliopsoas abscess is defined by direct dissemination of inflammation from the adjacent inflammatory organs, mainly from past spinal caries. Today, secondary iliopsoas results mainly from gastrointestinal diseases such as Crohn disease, appendicitis, ulcerative colitis, diverticulitis, colorectal cancer, or vertebral osteomyelitis, and only rarely from urologic diseases.

Streptococcus sanguis comprises part of the normal flora of the human oral cavity. Although it has been recognized increasingly as an important pathogen of endocarditis, $S$. sanguis is a rare cause of urinary tract infection. Anatomically, the iliopsoas muscle tends to be affected by infection of adjacent urologic organs. Streptococcus sanguis is a rare cause of psoas abscess, and to our knowledge, this infection has been reported previously only once [1]. We report a case of iliopsoas abscess caused by urinary tract $S$. sanguis infection.

\section{Case Presentation}

An 81-year-old male presented to the emergency department with complaints of pain in the right side of his back and disturbance in gait at the end of February 2016. Because he had fallen three times while walking several weeks prior to his visit, he had difficulty walking unassisted. He felt a dull pain in the right side of his back while walking and experienced frequent diarrhea after meals and cloudy urine. He was

\footnotetext{
${ }^{1}$ Department of Internal Medicine, Kobe Tokushukai Hospital, Tarumi, Kobe, Japan.

${ }^{2}$ Department of Critical Care and Emergency Medicine, Okayama University Hospital, Okayama, Japan.

(C) Nobuhiro Takeuchi et al. 2016; Published by Mary Ann Liebert, Inc. This Open Access article is distributed under the terms of the Creative Commons License (http://creativecommons.org/licenses/by/4.0), which permits unrestricted use, distribution, and reproduction in any medium, provided the original work is properly credited.
} 
apparently well until the onset of these symptoms. He had no history of diabetes mellitus or orthopedic diseases. $\mathrm{He}$ smoked 20 cigarettes and drank one bottle of whiskey per day. He was admitted to our hospital for further examination.

On admission, the patient's blood pressure was $136 / 51 \mathrm{~mm}$ $\mathrm{Hg}$, heart rate was 77 beats per minute with regular rhythm, body temperature was $37.2^{\circ} \mathrm{C}$, and blood oxygen saturation was $97 \%$ under atmospheric conditions. On clinical examination, his weight was $38 \mathrm{~kg}$, height was $153 \mathrm{~cm}$, and body mass index was $16.2 \mathrm{~kg} / \mathrm{m}^{2}$. Severe anemia was evident from palpebral conjunctiva pallor, but jaundice was not apparent from his bulbar conjunctiva. His abdomen was soft and flat with normal peristalsis; no mass or signs of peritoneal irritation were observed over the abdomen. Constovertebral angle tenderness was slightly evident in his back.

Blood chemistry analyses revealed severe anemia $\left(296 \times 10^{4} /\right.$ $\mathrm{mcL}$ red blood cells per microliter, hemoglobin $6.5 \mathrm{~g} / \mathrm{dL}$, hematocrit $21.3 \%$ ); and moderately decreased renal function (serum creatinine concentrations; $3.7 \mathrm{~g} / \mathrm{dL}$, blood urea nitrogen; $34.6 \mathrm{mg} / \mathrm{dL})$. Furthermore, mildly decreased total proteins $(6.4 \mathrm{~g} / \mathrm{dL})$, severely decreased albumin concentrations
( $1.8 \mathrm{~g} / \mathrm{dL})$, mildly elevated alkaline phosphatase (381 IU/L), and mildly elevated C-reactive proteins were also found.

Non-contrast computed tomography (CT) revealed right hydronephrosis and swollen iliopsoas muscle. Ultrasonography revealed a $7.7 \mathrm{~mm}$ stone and a distended right ureter that was affected by a $42.0 \times 29.2 \mathrm{~mm}$ low-echoic lesion in the iliopsoas muscle. Iliopsoas abscess was suspected based on these imaging results. There was no evidence on magnetic resonance imaging (MRI) of pyogenic spondylitis. Cardiac ultrasonography detected no vegetation in the chambers.

Urine cultures were found to contain Streptococcus sanguis that was sensitive to ampicillin and sulbactam. Then, administration of an antibacterial antibiotic (ampicillin and sulbactam $6 \mathrm{~g} / \mathrm{d}$ ) was initiated. Contrast-enhanced CT was performed on day five, revealing the connection between the right ureter and the iliopsoas abscess (Fig. 1A and 1B). On day eight, the abscess was drained by the percutaneous needle method under ultrasonography and a 7F pigtail catheter was placed successfully in the abscess cavity. Radiography revealed the communication between the abscess cavity and right-sided ureter (Fig. 1C). Abscess cultures contained

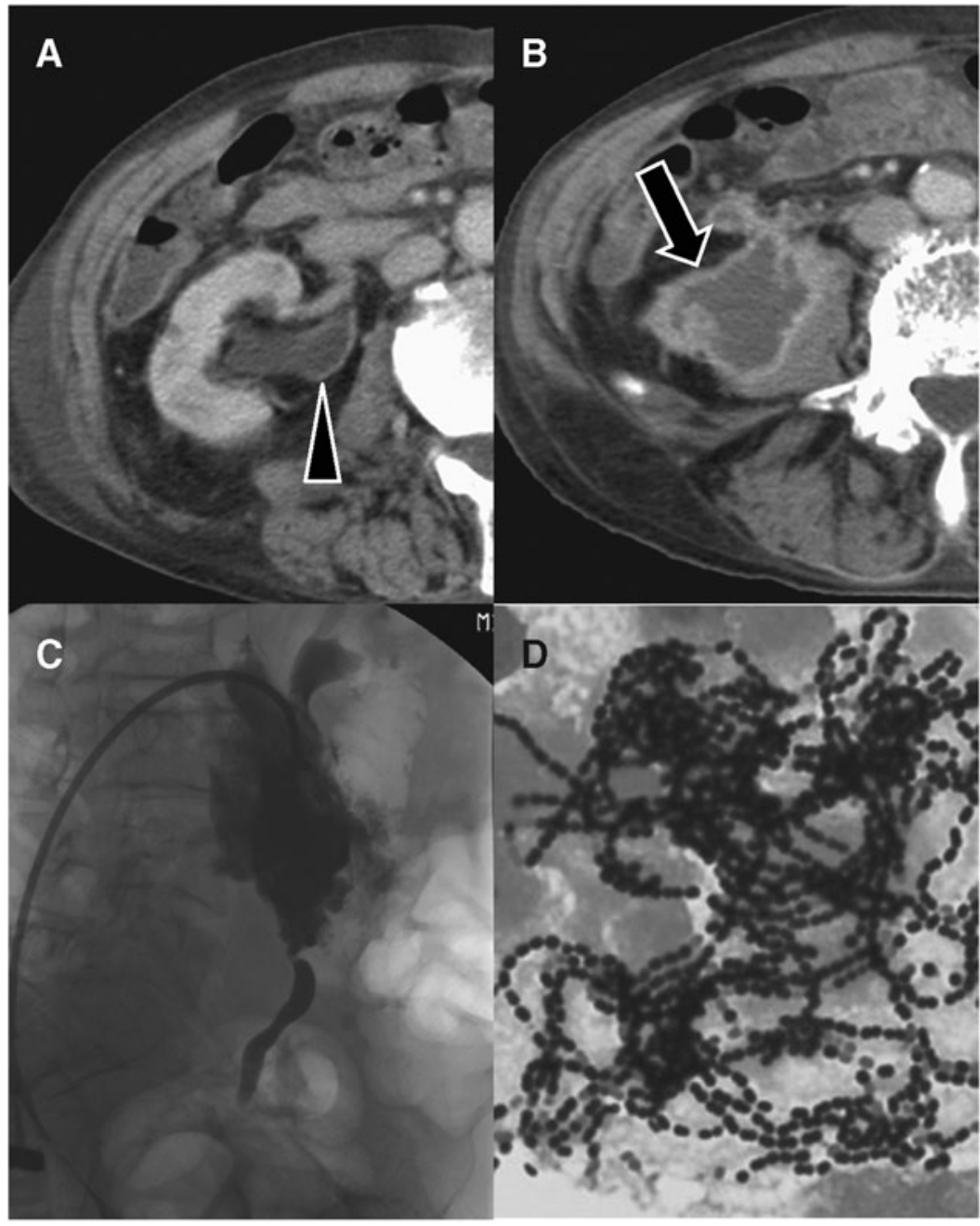

FIG. 1. Contrast-enhanced computed tomography revealed hydronephrosis in the right kidney with fat straddling the peritoneal space (arrowhead, A) and the low density cavity lined with contrast-enhanced margin, suggesting iliopsoas abscess (arrow, B). Imaging after ultrasonography-guided drainage of the abscess revealed the communication between the iliopsoas abscess cavity and dilated ureteropelvic system (C). Cultures of the abscess demonstrated Streptococcus sanguis (D). 
S. sanguis (Fig. 1D). Fever and inflammatory reaction improved after drainage and antibiotic treatment. On day 31, contrast radiography of the abscess revealed a reduction of the cavity and indicated that it no longer affected the ureter. Clinical course was uneventful and the patient was transferred to a rehabilitation facility.

\section{Discussion and Literature Review}

Streptococcus sanguis is a member of the viridans group of streptococci, which has been recognized increasingly as a pathogen of endocarditis and prosthetic joint infections. Streptococcus sanguis does not have a propensity to create parenchymatous abscesses and has not been recognized as a specific pathogen of iliopsoas abscess.

Iliopsoas abscess is becoming uncommon as a result of the progression of antibacterial therapy and the improvement of sanitary and nutritional conditions. However, the number of patients with iliopsoas abscess has been increasing as a result of the development of imaging modalities, including CT, MRI, and ultrasonography [2]. The underlying conditions of iliopsoas abscess include diabetes mellitus, aging, long-term administration of steroidal drugs, heavy drinking, and drug abuse. Our patient did not have a history of diabetes mellitus or steroidal drug use, however, his aging and lifestyle factors including alcohol abuse and malnutrition may have created a vulnerable immune system, which led to hematogenous spread of normal inhabitant resident microbiota from the oral cavity and formation of abscess in the iliopsoas muscle. Presumably, persistent urinary tract infection and inflammation associated with the ureter stone might contribute to communication between the urinary tract and the iliopsoas muscle.

Clinical presentation of iliopsoas includes back pain, limp, and fever. However, only $30 \%$ of patients with iliopsoas abscess present this triad of symptoms [3]. Many other patients with iliopsoas abscess visit hospitals with atypical symptoms, including deterioration of consciousness, coma, or appetite loss, therefore, the diagnosis of iliopsoas abscess is sometimes challenging and only found through medical interview or physical examination. Although conservative treatment of iliopsoas abscess is possible with the use of broad-spectrum and highly efficient antibacterial drugs, open drainage has been a mainstay for the treatment of iliopsoas abscess. The efficiency and less invasive nature of percutaneous drainage of iliopsoas abscess under ultrasonography or CT are proposed [4]. In our case, percutaneous drainage was useful, not only for drainage but also for detecting the communication between the ureter and abscess. These appropriate strategies yielded an uneventful recovery of the patient.

Previous literature has reported cases of iliopsoas abscess after urologic interventions for compacted ureteral stones, including extracorporeal shock wave lithotripsy (ESWL) and retroperitoneoscopic ureterolithotomy (RPU) [5-7]. Although a rare complication, clinicians should be aware that urologic intervention can cause formation of an abscess in the iliopsoas muscle.

\section{Conclusion}

It is important to recognize $S$. sanguis as a previously unrecognized species of Streptococcus in the etiology of iliopsoas abscess and to be aware of its predisposing factors. Sharing our experience with readers may help clinicians make the proper selection of antimicrobial agents, a key to the successful management of iliopsoas abscess.

\section{Acknowledgments}

No funding support was given for this study.

\section{Author Disclosure Statement}

No competing financial interests exist.

\section{References}

1. dos Santos VM, Fachinelli LR, Farage L, et al. An 81-yearold male with iliopsoas abscess by Streptococcus sanguis. Infez Med 2015;23:56-60.

2. Chern $\mathrm{CH}, \mathrm{Hu}$ SC, Kao WF, et al. Psoas abscess: Making an early diagnosis in the ED. Am J Emerg Med 1997;15:83-88.

3. Suzuki K, Yamaguchi T, Iwashita Y, et al. Case series of iliopsoas abscesses treated at a university hospital in Japan: Epidemiology, clinical manifestations, diagnosis and treatment. Intern Med 2015;54:2147-2153.

4. Kang M, Gupta S, Gulati M, et al. Ilio-psoas abscess in the paediatric population: Treatment by US-guided percutaneous drainage. Pediatr Radiol 1998;28:478-481.

5. Langille GM, Norman RW. Psoas abscess from ureteric stone perforation. Can J Urol 2010;17:5408-5410.

6. Komori K, Yamamoto K, Momohara C, et al. A case of psoas abscess with ureteral stone. Hinyokika Kiyo 2003;49:25-27.

7. Kiyota H, Ikemoto I, Asano K, et al. Retroperitoneoscopic ureterolithotomy for impacted ureteral stone. Int J Urol 2001; 8:391-397.

Address correspondence to: Dr. Atsunori Nakao

Department of Critical Care and Emergency Medicine Okayama University Graduate School of Medicine Dentistry and Pharmaceutical Sciences 2-5-1 Shikata-cho

Kita-ku, Okayama-shi Okayama, 700-8558 Japan

E-mail: qq-nakao@okayama-u.ac.jp

Abbreviations Used
$\mathrm{CT}=$ computed tomography
$\mathrm{ESWL}=$ extracorporeal shock wave lithotripsy
$\mathrm{MRI}=$ magnetic resonance imaging
$\mathrm{RPU}=$ retroperitoneoscopic ureterolithotomy

Cite this article as: Takeuchi N, Emori $\mathrm{K}$, Yoshitani M, Soneda J, Naito H, Yumoto T, Tsukahara K, Nakao A (2016) A successfully treated case of iliopsoas abscess affecting urinary tract infection caused by Streptococcus sanguis. Surgical Infections Case Reports 1:1, 123-125, DOI: $10.1089 / \mathrm{crsi} .2016 .0032$ 